\title{
Prediction of pre-eclampsia in early pregnancy using PAPP-A and uterine artery pulsatility index
}

\author{
Charu Pratap*, Srishti Thakur
}

Department of Obstetrics and Gynecology, Subharti Medical College, Dehradun, Uttarakhand, India

Received: 26 September 2018

Accepted: 22 October 2018

*Correspondence:

Dr. Charu Pratap,

E-mail: charupratap30@gmail.com

Copyright: (C) the author(s), publisher and licensee Medip Academy. This is an open-access article distributed under the terms of the Creative Commons Attribution Non-Commercial License, which permits unrestricted non-commercial use, distribution, and reproduction in any medium, provided the original work is properly cited.

\begin{abstract}
Background: The aim is to study the role of first trimester pregnancy associated plasma protein A (PAPP A) and second trimester uterine artery pulsatility index (PI) in prediction of pre-eclampsia in early pregnancy.

Methods: This prospective study conducted at GSVM Medical College, Kanpur from May 2011 to May 2012 included 100 pregnant women. Serum PAPP-A level was determined in the first trimester (8-14 weeks) and uterine artery Doppler was done at 20-25 weeks of gestation.

Results: Out of 100 patients 7 developed pre-eclampsia. Mean PAPP-A level in pre eclamptic and normotensive patients were $1.25 \pm 1.4$ and $2.5 \pm 0.5 \mathrm{ng} / \mathrm{ml}$ respectively. Mean PI level in pre eclamptic and normotensive patients were $1.77 \pm 0.28$ and $0.96 \pm 0.25$ respectively. The sensitivity and specificity of PAPP-A at cut off $<1.7 \mathrm{ng} / \mathrm{ml}$ was $71 \%, 71 \%$ respectively. The sensitivity and specificity of pulsatility index at cut off $>1.4$ was $100 \%, 94 \%$ respectively.

Conclusions: Low level of maternal serum PAPP-A at 8-14weeks and high level of uterine artery PI at 22-25 weeks of pregnancy can predict subsequent development of pre-eclampsia.
\end{abstract}

Keywords: Early pregnancy, PAPP-A, Pre-eclampsia, Uterine artery pulsatility index

\section{INTRODUCTION}

Pre-eclampsia affects approximately 3-5\% of pregnancies and is a significant cause of maternal and fetal morbidity and mortality. It is defined as the occurrence of hypertension and proteinuria during pregnancy. ${ }^{1}$ The clinical syndrome of PE arises in the second half of pregnancy. However, many underlying factors including defective placentation may already be apparent in the first and early second trimester in many patients. Poor placentation in early pregnancy causes excessive maternal systemic inflammatory response leading to preeclampsia. $^{2}$

Early identification of high-risk pregnancy may facilitate the development of new strategies for antenatal surveillance or prevention and thus improve maternal and perinatal outcome. A combination of clinical characteristics and first trimester maternal serum biomarkers provides an accurate screening for early onset preeclampsia. ${ }^{4}$ The purpose of present study was to evaluate the role of PAPP-A and uterine artery pulsatility index (PI) in predicting pre-eclampsia in early pregnancy. First trimester maternal serum PAPP-A is reduced in pregnancies with pre-eclampsia. ${ }^{3}$ With more severe or early pre-eclampsia the PAPP-A levels may be further reduced. PAPP-A is a protease for IGF binding protein (IGFBP-4). IGFBP-4 bind to IGF-I and IGF- II, inhibiting their interaction with cell surface receptors. PAPPA-A breaks down IGFBP, so low level of PAPP-A would be expected to be associated with high level of IGF-BP and therefore low level of IGF. The IGFs have key role in regulating fetal growth. The IGF increases uptake of glucose and amino-acids in cultured trophoblast 
and have an important role in autoimmune and paracrine control of trophoblast invasion of deciduas.

Doppler ultrasound is a non-invasive technique, which can easily be used for evaluation of maternal and fetal hemodynamics. In pre-eclampsia, defective placentation leads to inadequate trophoblastic invasion and decreased uterine perfusion. Uterine arteries (UA) are the main arteries for blood supply of the pregnant uterus. They are believed to reflect the decrement in impedance to flow in spiral arteries during trophoblastic invasion. Doppler studies of uterine arteries in the second trimester of pregnancy have reported that increased pulsatility index or the presence of an early diastolic notch identifies women at risk of developing pre-eclampsia. ${ }^{5,7}$ Several studies have shown that a combination of maternal history, low PAPP A level and abnormal uterine artery Doppler is a better predictor of pre-eclampsia in pregnancy than either test alone. ${ }^{6-9}$

\section{METHODS}

The present study was conducted on 100 pregnant women attending outpatient department and indoor cases of Upper India Sugar Exchange Maternity Hospital, Department of Obstetrics and Gynecology G.S.V.M. Medical College, Kanpur, over a period of 12 months during the year May 2011 to 2012. Taking the incidence of pre-eclampsia in pregnancy to be $5 \%$, the sample size was calculated using the formula $(4 \mathrm{pq} / 25)$, where $\mathrm{p}$ is the incidence and $\mathrm{q}=(1-\mathrm{p})$, taking $\mathrm{d}=5$, i.e. a $20 \%$ margin of allowable error. The sample size came out to be 76 . Rounding off and allowing for the margin and loss of follow up it was decided to carry the study on 100 patients. The study design was blind prospective study. It included antenatal patients with gestational age between 11-24 weeks.

Primi as well as multipara with singleton pregnancies were included. Women with chronic hypertension, diabetes, chronic renal disease or with history of hypertension, proteinuria, pre-eclampsia in previous pregnancies were excluded from the present study. A detailed history was obtained, thorough examination was done, and routine antenatal investigations were carried out.

Special investigations were-Uterine artery Doppler Both uterine arteries were identified on color Doppler imaging and pulsed wave Doppler was used to obtain 3 sequential good waveforms. Flow velocity waveform were stored and analyzed. Pulsatility indices were calculated immediately.

Collection of blood sample for PAPP-A after the patient has been selected for study, after taking informed consent, $10 \mathrm{ml}$ blood sample was taken by disposable syringe by vene-puncture under all aseptic precautions.

Care was taken to avoid haemolysis and the sample was allowed to clot at room temperature. Collected samples is centrifuged for 25 minutes at $5000 \mathrm{rpm}$. Samples were taken at 11-14 weeks for PAPP A. These pregnant patients were followed till delivery. In all studied participants serum PAPP A was measured using ELISA. Pre-eclampsia was defined according to the guidelines of the International Society for the Study of Hypertension in Pregnancy.

\section{Statistical analysis}

Appropriate statistical tools like students t-test, chisquare tests and ROC curve analysis have been employed using statistical softwares like SPSS and medcalc.

\section{RESULTS}

Out of 100 patients, only $70 \%$ of patients could be followed up to delivery. 7 patients in study group developed pre-eclampsia. 6 patients had missed abortion and 24 patients could not be followed up to delivery (Table 1).

Table 1: Number of cases.

\begin{tabular}{|llllll|}
\hline Total No. of patients & Missed abortion & Dropped out cases & $\begin{array}{l}\text { Total no. of } \\
\text { patients followed } \\
\text { up to delivery }\end{array}$ & Pre-eclampsia & Normotensive \\
\cline { 2 - 4 } & 6 & 24 & 70 & 7 & 63 \\
\hline Percentage & 6 & 24 & 70 & 7 & 63 \\
\hline
\end{tabular}

The mean age in pre eclamptic group is $24.4 \pm 4.86$ and normotensive group is $23 \pm 4.12$ respectively, with majority of women in 19-22-year age group.

No significant difference was noted in the mean age of both the groups. In present study majority of the women were nullipara in both the normotensive and in the pre eclamptic group.

$57.14 \%$ of the preeclamptic patients and $53.96 \%$ of the normotensive women were primigravida. 


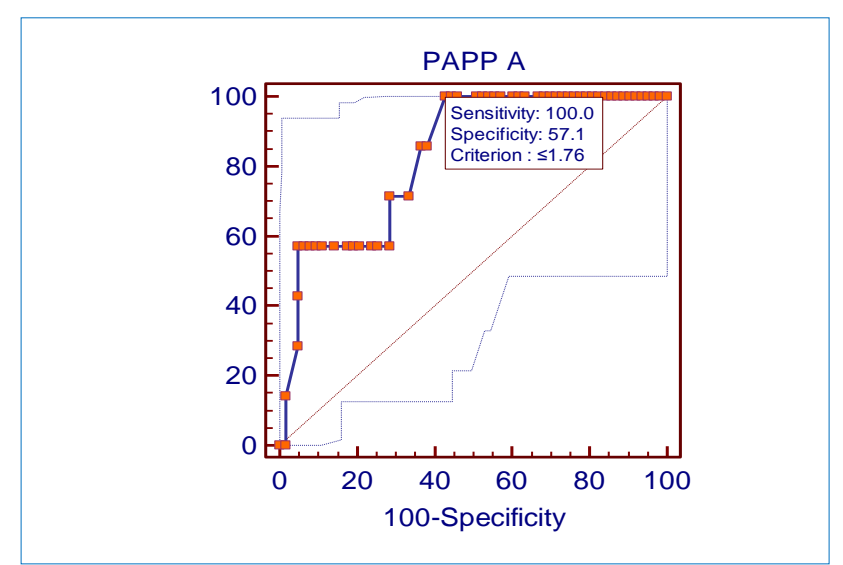

Figure 1: ROC curve for PAPP A: to assess the discriminatory power of PAPP A as a diagnostic test for pre-eclampsia, roc curve analysis was performed.

In my study, maximum number of women belong to low socio-economic status $0.71 .42 \%$ among pre eclamptic and $63.49 \%$ among normotensive women belonged to low socioeconomic status. In present study, in pregnancies with pre-eclampsia, maternal PAPP A level was reduced in first trimester (PAPP A<1.76).

ROC curve analysis was used to determine the cut off value of PAPP A to predict pre-eclampsia (Figure 1). At cut off of $<1.7$, the sensitivity is $71.43 \%$ and specificity is $71.43 \%$ Table 2)

Table 2: ROC curve for PAPP A.

\begin{tabular}{|c|c|}
\hline Variable & PAPP A \\
\hline Classification variable & PreE \\
\hline Sample size & 70 \\
\hline Positive group: & 7 \\
\hline Negative group: & 63 \\
\hline Disease prevalence $(\%)$ & unknown \\
\hline
\end{tabular}

Mean PAPP A level among pre eclamptic group was $1.2529 \pm 1.406$ whereas mean PAPP A among normotensive group was $2.5013 \pm 0.45(95 \%$ confidence interval being $0.74088-1.75594)$. The $\mathrm{t}$ value was 5.08 and $\mathrm{p}$ value was $<0.0001$ which is statistically significant (Table 4) (Table 5).

Table 3: Comparison of PAPP A levels between pre-eclamptic and normotensive cases.

\begin{tabular}{|llllllll|} 
Variable & Pre-eclamptic & Normotensive & t value & P value & Mean difference & $95 \%$ C.I. of mean difference \\
\hline PAPP A & $1.2529 \pm 1.41$ & $2.5013 \pm 0.45$ & 5.08 & 0.001 & 1.24841 & 0.74088 & 1.75594 \\
\hline
\end{tabular}

Table 4: Comparison of uterine artery PI levels between pre-eclamptic and normotensive cases.

\begin{tabular}{|llllllll} 
Variable & Pre-eclamptic & Normotensive & t value & P value & Mean difference & 95\% C.I. of mean difference \\
\hline Mean PI & $1.7771 \pm 0.28$ & $0.9627 \pm 0.25$ & 8.179 & 0.0001 & 0.8145 & 0.7408 & 1.7559
\end{tabular}

Table 5: Criterion values and coordinates of the ROC curve.

\begin{tabular}{|lllllll|}
\hline Criterion & Sensitivity & $95 \%$ CI & Specificity & $95 \%$ CI & +LR & -LR \\
\hline$<0.85$ & 0.00 & $0.0-41.0$ & 100.00 & $94.3-100.0$ & & 1.00 \\
\hline$\leq 0.85$ & 0.00 & $0.0-41.0$ & 98.41 & $91.5-100.0$ & 0.00 & 1.02 \\
\hline$\leq 0.87$ & 14.29 & $0.4-57.9$ & 98.41 & $91.5-100.0$ & 9.00 & 0.87 \\
\hline$\leq 0.88$ & 28.57 & $3.7-71.0$ & 95.24 & $86.7-99.0$ & 6.00 & 0.75 \\
$\leq 0.92$ & 57.14 & $18.4-90.1$ & 95.24 & $86.7-99.0$ & 12.00 & 0.45 \\
\hline$\leq 1.68$ & 57.14 & $18.4-90.1$ & 71.43 & $58.7-82.1$ & 2.00 & 0.60 \\
\hline$\leq 1.7$ & 71.43 & $29.0-96.3$ & 71.43 & $58.7-82.1$ & 2.50 & 0.40 \\
\hline$\leq 1.72$ & 71.43 & $29.0-96.3$ & 66.67 & $53.7-78.0$ & 2.14 & 0.43 \\
\hline$\leq 1.74$ & 85.71 & $42.1-99.6$ & 63.49 & $50.4-75.3$ & 2.35 & 0.23 \\
\hline$\leq 1.75$ & 85.71 & $42.1-99.6$ & 61.90 & $48.8-73.9$ & 2.25 & 0.23 \\
\hline$\leq 1.76$ & 100.00 & $59.0-100.0$ & 57.14 & $44.0-69.5$ & 2.33 & 0.00 \\
\hline$\leq 6.75$ & 100.00 & $59.0-100.0$ & 0.00 & $0.0-5.7$ & 1.00 & \\
\hline
\end{tabular}

'p' value less than 0.0001 statistically significant

The area under the curve (AUC) for PAPP A is 0.831 (95\% confidence interval- 0.723 to 0.91 ), $\mathrm{p}$ value $<0.001$ (Table 6). Lower boundary of $95 \%$ confidence curve of AUC is below the diagonal line (0.50), therefore PAPP A alone cannot be claimed as an ideal discriminator. In present study, mean uterine artery pulsatility index is increased in pregnancies developing preeclampsia. Mean PI in pregnancies developing pre-eclampsia was $1.7771 \pm 0.280$ and mean PI in normotensive group was $0.9627 \pm 0.247$. $95 \%$ confidence interval for mean 
difference being $0.74088 \pm 1.75594$. The difference of uterine artery PI levels between the two groups was found to be statistically highly significant $(\mathrm{p}<0.0001)$ (Table 3).

Table 6: Area under the ROC curve (AUC).

\begin{tabular}{|ll|}
\hline Area under the ROC curve (AUC) & 0.831 \\
\hline Standard error & 0.0710 \\
\hline $95 \%$ Confidence interval $^{\mathrm{b}}$ & 0.723 to 0.910 \\
\hline z statistic & 4.662 \\
\hline Significance level P (Area=0.5) & $<0.0001$ \\
\hline
\end{tabular}

The area under the curve for uterine artery pulsatility index (PI) is 0.984 (95\% confidence interval of 0.920 0.999 ) and $p$ value is $<0.0001$ (Table 6). This shows that the association is statistically highly significant (Figure 2).

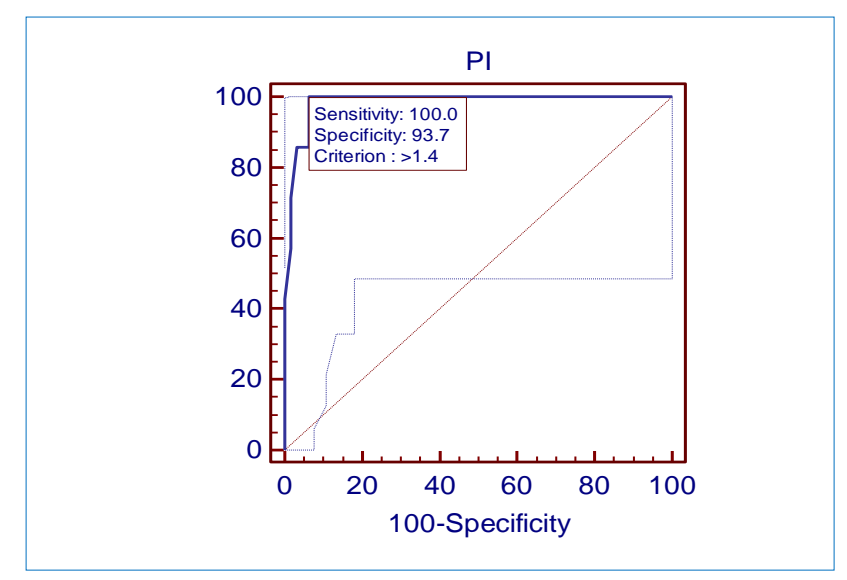

Figure 2: ROC curve for pulsatility index (PI): To assess the discriminatory power of pulsatility index as a diagnostic test for pre-eclampsia, ROC curve analysis was performed.

ROC curve analysis was used to determine the cut off value of uterine artery pulsatility index (PI) to predict pre-eclampsia (Table 7) (Table 8).

Table 7: ROC curve for PAPP A.

\begin{tabular}{|l|ll|}
\hline \multicolumn{2}{|l}{ Variable } & \multicolumn{2}{l}{ PI } \\
\hline Classification variable & & 70 \\
\hline Sample size & PreE $=1$ & 7 \\
\hline Positive group: & PreE $=0$ & 63 \\
\hline Negative group: & unknown \\
\hline Disease prevalence $(\%)$ & unk
\end{tabular}

Table 8: Area under the ROC curve (AUC).

\begin{tabular}{|c|c|}
\hline Area under the ROC curve (AUC) & 0.984 \\
\hline $\begin{array}{l}95 \% \text { Confidence interval of area } \\
\text { under curve }\end{array}$ & 0.920 to 0.999 \\
\hline Significance level P (Area=0.5) & $<0.0001$ \\
\hline
\end{tabular}

At cut off of $>0.4$, sensitivity is $100 \%$ and specificity is $93.7 \%$
At cut off of $>1.4$, the sensitivity is $100 \%$ and specificity is 93.65 (Table 9). In present study we found that patients who had elevated uterine artery pulsatility index in second trimester developed pre-eclampsia subsequently on follow up.

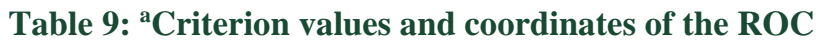
curve.

\begin{tabular}{lllll|}
\hline Criterion & Sensitivity & Speciffeity & +LR & -LR \\
\hline$\geq 0.62$ & 100.00 & 0.00 & 1.00 & \\
\hline$>1.4$ & 100.00 & 93.65 & 15.75 & 0.00 \\
\hline$>1.42$ & 85.71 & 93.65 & 13.50 & 0.15 \\
\hline$>1.52$ & 85.71 & 96.83 & 27.00 & 0.15 \\
\hline$>1.6$ & 71.43 & 98.41 & 45.00 & 0.29 \\
\hline$>1.62$ & 57.14 & 98.41 & 36.00 & 0.44 \\
\hline$>1.7$ & 42.86 & 100.00 & & 0.57 \\
\hline$>2.2$ & 0.00 & 100.00 & & 1.00 \\
\hline $\begin{array}{l}\text { p value is }<0.0001 . \\
\text { significant. This }\end{array}$ & & & & \\
\hline
\end{tabular}

Thus, ROC curve analysis for pre eclamptic cases Vs normotensives yielded areas under curve for PAPP A and PI respectively of $0.831(95 \% \mathrm{CI}, 0.723$ to $0.9 ; \mathrm{p}<0.001)$ and 0.984 (95\% CI,0.920-0.999; $\mathrm{p}<0.0001)$. At a specificity set to $80 \%$, the sensitivities were $57.14 \%$ and $100 \%$ for PAPP A and PI respectively in pre eclamptic cases.

\section{DISCUSSION}

In present study PAPP A levels in first trimester were reduced in patients who developed pre-eclampsia. Contrary to present study PAPP A levels were found to be increased in pre-eclampsia in a study by Bersinger NA et al2. But the results of present study are well corroborated with various other studies.

Poon et al showed that PAPP A levels were significantly lower among those women who developed pre-eclampsia and PAPP A related patient specific risk for preeclampsia can be modified by the measurement of uterine artery $\mathrm{PI} .^{3}$

In a study by $\mathrm{F}$. Audibert et al4, considering $10 \%$ of false positive, the sensitivity of PAPP A serum levels was $35.2 \%$ and $55.6 \%$ in the diagnosis of pre-eclampsia and early preeclampsia.

In present study we found that patients who had elevated uterine artery pulsatility index in second trimester developed pre-eclampsia later in pregnancy. Present study is supported by the following. Papageorghiou et al have studied the Doppler velocimetry of maternal uterine arteries between the 22nd and 24th weeks of gestation by the measurement of PI. ${ }^{5}$

The mean PI in patients that developed preeclampsia was more than that of patients who did not develop preeclampsia. In a similar study Pilalis A, Souka AP et al, 
concluded that the combination of maternal history with abnormal uterine artery Doppler and low PAPP-A level at 11-14 weeks achieves better results than does either test alone in the prediction of pre-eclampsia and SGA. ${ }^{6}$

Cnossens Js, Morris RK, ter Riet G et al concluded that abnormal uterine artery waveforms are a predictor of preeclampsia. $^{7}$

An increased uterine artery pulsatility index, alone or combined with notching, is the most predictive Doppler index. The results of present study well corroborate with following studies.

Spencer K, Yu CK, Cowans NJ et al8, found that in the pregnancies resulting in pre-eclampsia and FGR, the median PAPP-A was lower (0.844 and $0.813 \mathrm{MoM})$ and the median uterine artery mean PI was higher (1.56 and 1.18).

They concluded that the combination of first trimester serum PAPP-A and uterine artery mean PI at 22-24 weeks improves the screening efficacy for the prediction of pre-eclampsia. Spencer K, Cowans NJ et al concluded that first-trimester PAPP-A provides some prediction for pre-eclamspia when combined with PI. $^{9}$ Odibo AO, Zhong $\mathrm{Y}$ et al conducted study and concluded that for a fixed false positive rate (FPR) of $20 \%$, PAPP-A and mean uterine artery pulsatility index identified $58 \%$ and $62 \%$, respectively, of women who developed any form of pre-eclampsia. ${ }^{10}$

\section{CONCLUSION}

We conclude that PAPP A and PI are predictors of women at risk to develop preeclampsia. Out of these, uterine artery pulsatility index is the better predictor. PAPP-A is also a relative predictive factor of preeclampsia, but its discriminatory value is not as good as uterine artery PI. But uterine artery PI is a late predictor.

The advantage of PAPP-A is that it is an earlier indicator of pre-eclampsia and it will help in selecting patients who are prone for development of pre-eclampsia around 10 weeks earlier and thus help us in detecting high risk cases very early in gestation when the pathological changes begin.

Thus, according to present study, it is possible that measuring of PAPP-A in the first trimester and uterine artery pulsatility index in second trimester can be used for prediction of pre-eclampsia.

This will make it possible to implement appropriate preventive and treatment strategies by better prediction of preeclampsia and thereby reducing maternal and perinatal mortality. Based on present study, it is recommended to design further multicenter studies with more sample size to establish a better screen protocol of patients with preeclampsia.

\section{ACKNOWLEDGMENTS}

Authors would like to thank to the patients and hospital for their cooperation.

Funding: No funding sources

Conflict of interest: None declared

Ethical approval: The study was approved by the Institutional Ethics Committee

\section{REFERENCES}

1. Roberts JM, Cooper DW.Pathogenesis and genetics of preeclampsia. Lancet 2001;357(9249):53-6.

2. N.A. Bersinger, A.K. Smarason, S. Muttukrishna, N.P. Groome, and C.W. Redman. Women with preeclampsia have increased serum levels of pregnancy associated plasma protein A (PAPP-A), inhibin A, activin $\mathrm{A}$ and Soluble E-selectin. Hyperten Preg. 2003;22(1):45-55.

3. Poon LC, Maiz N, Valencia C, Plasencia W, Nicolaides KH. First-trimester maternal serum pregnancy-associated plasma protein-A and preeclampsia. Ultrasound

Obstet Gynecol.2009;33(1):23-33.

4. Audibert F, Boucoiran I, An N, Aleksandrov N, Delvin E, Bujold E et al. Screening for preeclampsia using first-trimester serum markers and uterine artery Doppler in nulliparous women. Am J Obstet Gynecol. 2010;203(4):383-e1.

5. Papageorghiou AT, Yu CK, Erasmus IE, Cuckle HS, Nicolaides KH. Assessment of risk for the development of pre-eclampsia by maternal characteristics and uterine artery Doppler. BJOG. 2005;112(6):703-9.

6. Pilalis A, Souka AP, Antsaklis P, Daskalakis G, Papantoniou N, Mesogitis S et al.Screening for preeclampsia and fetal growth restriction by uterine artery Doppler and PAPP-A at 11-14 weeks' gestation.Ultrasound Obstet Gynecol. 2007;29(2):135-40.

7. Cnossen JS, Morris RK, ter Riet G, Mol BW, van der Post JA, Coomarasamy A et al. Use of uterine artery Doppler ultrasonography to predict pre-eclampsia and intrauterine growth restriction: a systematic review and bivariable meta-analysis. Canadian Med Associat J. 2008;178(6):701-11.

8. Spencer K, Yu CK, Cowans NJ, Otigbah C, Nicolaides KH. Prediction of pregnancy complications by first-trimester maternal serum PAPP-A and free beta-hCG and with secondtrimester uterine artery Doppler. Prenat Diagn. 2005;25(10):949-53

9. Spencer K, Cowans NJ, Chefetz I, Tal J, Meiri H. First-trimester maternal serum PP-13, PAPP-A and second-trimester uterine artery Doppler pulsatility 
index as markers of pre-eclampsia. Ultrasound in Obstetrics and Gynecology. 2007;29(2):128-34.

10. Odibo AO, Zhong Y, Goetzinger KR, Odibo L, Bick JL, Bower CR et al. First-trimester placental protein 13, PAPP-A, uterine artery Doppler and maternal characteristics in the prediction of pre-eclampsia. Placenta. 2011;32(8):598-602.
Cite this article as: Pratap C, Thakur S. Fetal

Prediction of pre-eclampsia in early pregnancy using PAPP-A and uterine artery pulsatility index. Int $\mathbf{J}$

Reprod Contracept Obstet Gynecol 2018;7:5005-10. 